\title{
SITUAÇÃO DAS BIBLIOTECAS ESCOLARES CONTEMPLADAS PELO PROGRAMA MANUEL BANDEIRA DE FORMAÇÃO DE LEITORES
}

\author{
Aline Rafaela Silva dos Anjos ${ }^{1}$ \\ Stela Tavares da Silva Brandão ${ }^{2}$ \\ Ester Calland de Sousa Rosa ${ }^{3}$
}

\begin{abstract}
RESUMO: A biblioteca escolar (BE) é entendida no presente trabalho como um espaço de aquisição de conhecimento, e para a realização da pesquisa, baseou-se em indicadores de qualidade desse espaço a partir de estudos anteriores. A pesquisa teve como objetivo geral, verificar a situação das 58 BEs do Recife, contempladas pelo Programa Manuel Bandeira de Formação de Leitores. Para tanto, foram mapeadas quais eram as escolas que tinham a BE em funcionamento. Definidas estas, foi realizada uma regionalização para que cobrisse toda a cidade, a partir disso, houve a aplicação de questionários e observação das 16 BEs, além de entrevista com pessoas envolvidas no Programa. Como resultado principal, percebeu-se que havia uma discrepância entre o que a escola anunciava e o que foi constatado. Conclui-se que a política de implantação e reestruturação de BEs sofreu descontinuidade.
\end{abstract}

Palavras-chave: Biblioteca Escolar do Recife. Programa Manuel Bandeira de Formação de Leitores. Políticas públicas.

\section{SITUATION OF THE SCHOOL LIBRARIES CONTEMPLATED BY THE MANUEL BANDEIRA PROGRAM FOR} READING TRAINING

\begin{abstract}
The school library (BE) is understood in the present work as a space for acquiring knowledge, and for carrying out the research, it was based on quality indicators of this space from previous studies. The research had as general objective, to verify the situation of the 58 BEs of Recife, contemplated by Manuel Bandeira Program of Training of Readers. In order to do so, the schools that had BE in operation were mapped. Once these were defined, a regionalization was carried out to cover the whole city. From this, questionnaires were applied and 16 BEs were observed, as well as interviews with people involved in the Program. As a main result, it was noticed that there was a discrepancy between what the school announced and what was found. It is concluded that the BEs implementation and restructuring policy suffered discontinuity.
\end{abstract}

KEYWORDS: Recife School Library. Manuel Bandeira Program for the Training of Readers. Public policy.

\section{INTRODUÇÃO}

O Programa Manuel Bandeira de Formação de Leitores (PMBFL) foi proposto e implantado como política pública pela Secretaria de Educação, Esporte e Lazer do Recife a partir de 2008. Uma das metas do Programa, foco desta investigação, foi a realização de um concurso para instalação ou reestruturação de Bibliotecas Escolares (BE) e que contemplou cinquenta e oito unidades escolares da rede municipal com recursos financeiros e apoio técnico.

Estudos evidenciam que a presença de uma BE possui impactos significativos na vida dos estudantes. De acordo com a International Federation of Library Associations and Institutions (IFLA) e Organização das Nações Unidas para a Educação, a Ciência e a Cultura (UNESCO) (2005, p.4): “A

\footnotetext{
1 Mestranda em Ensino das Ciências e Matemática pela Universidade Federal Rural de Pernambuco- UFRPE alinerafaela33@gmail.com

${ }^{2}$ Graduada em Pedagogia pela Universidade Federal de Pernambuco- UFPE-tavares.stela@gmail.com

${ }^{3}$ Doutora em Psicologia Escolar e Desenvolvimento Humano- Universidade de São Paulo- USP-esterosa2014@ gmail.com
} 
biblioteca escolar habilita os alunos para a aprendizagem ao longo da vida e desenvolve sua imaginação, preparando-os para viver como cidadãos responsáveis".

Realizou-se um levantamento bibliográfico de pesquisas publicadas nos últimos cinco anos sobre a temática da BE com o objetivo de situar a temática no cenário acadêmico. Em periódicos da CAPES foram localizadas 89 pesquisas, no Banco de Teses e Dissertações do Brasil, foram mapeados 59 trabalhos e em repositórios institucionais de Universidades Públicas do Estado de Pernambuco foram encontrados apenas dois artigos. Para identificar os estudos anteriores a 2011, utilizou-se como referência uma pesquisa coordenada por Campello, que abrangeu o período de 1975 a 2011 e que estabeleceu o estado da arte da área de bibliotecas escolares (Campello, et al 2013). Além deste estudo, também recorreu-se a outra pesquisa de mesmo recorte de tempo, igualmente realizada por Campello e colaboradores (2012) que mapeou os estudos sobre a situação da BE no Brasil. Neste levantamento acerca das pesquisas que traziam um diagnóstico do funcionamento de BE no Brasil, evidenciou-se que na realidade brasileira, e mais particularmente na região Nordeste, poucas pesquisas investigaram o estado de funcionamento das BEs. Apesar deste limite, em tais estudos, foram localizadas teses, dissertações, artigos em periódicos e trabalhos de eventos em áreas como Educação, Ciências da Informação e Biblioteconomia, o que evidencia que se trata de um tema que é abordado a partir de diferentes campos de estudos.

Diante destas inquietações e das lacunas identificadas no campo a ser investigado, a presente pesquisa teve, como objetivo principal verificar a atual situação das cinquenta e oito bibliotecas escolares situadas na rede municipal do Recife, que foram contempladas pelo Programa Manuel Bandeira de Formação de Leitores (PMBFL) com instalação ou reestruturação, levando em consideração a investigação das condições do espaço físico (infraestrutura e condições de uso) das BEs contempladas pelo Programa que estavam em funcionamento; Identificação os recursos materiais (equipamentos e acervo) presentes nessas bibliotecas e avaliação se houve continuidade na política pública implantada através do PMBFL no que se refere à implantação e reestruturação de BEs.

Desta forma, apresentaremos a seguir as bases teóricas nas quais nos baseamos para esta investigação. Posteriormente apresentaremos a metodologia utilizada, as análises dos dados coletados e os resultados encontrados. 


\section{APARATO LEGAL, ORIENTAÇÕES GOVERNAMENTAIS E DIRETRIZES NO CAMPO DAS BILBIOTECAS ESCOLARES}

Apesar da fragilidade identificada no campo da pesquisa acerca da BE, é possível considerar que ao longo do tempo vai se delineando um aparato legal e de documentos norteadores de políticas públicas nesta área. Esta trajetória é objeto desta seção.

\subsection{PROGRAMA NACIONAL BIBLIOTECA DA ESCOLA (PNBE).}

Em 1997 houve a implementação do Programa Nacional Biblioteca da Escola (PNBE), que de acordo com o Fundo Nacional de Desenvolvimento da Educação (FNDE) busca prover as escolas de ensino público do país, contemplando todas as modalidades do ensino básico, com o fornecimento de obras e demais materiais de apoio à prática da educação básica. Segundo Paiva e Bereblum (2015), o PNBE com a sua política de distribuição de livros, foi o principal caminho adotado pelo governo federal enquanto política de formação de leitores nas escolas públicas. As autoras, no entanto, identificam limites nessa política, devido à falta de formação e tempo dos professores para utilização do acervo.

\subsection{DOCUMENTO BIBLIOTECA NA ESCOLA}

No ano de 2006, o Ministério da Educação (MEC) lançou um documento chamando "Biblioteca na Escola", que objetivou discutir com os professores e mediadores de leitura a função da escola de formar leitores. Em seu texto, apresenta questões sobre a formação da BE, a importância de se possuir diferentes gêneros, sugestões de trabalho e de obras. Sobre a organização do espaço físico, traça as seguintes características básicas: ser seco, arejado e também iluminado.

De acordo com Brasil (2006), o espaço de leitura precisa ser um ambiente agradável e atrativo para professores e alunos. Dessa forma percebe-se que o Ministério de Educação oferece, por meio deste documento, sugestões para a organização das bibliotecas escolares, privilegiando tanto os aspectos infraestruturas quanto de funcionamento. No entanto, não fica claro que apoio o Governo Federal daria a gestores de redes públicas e de escolas na implementação e manutenção de BEs. Ao que parece, o documento restringe-se a pontuar orientações, sem efetivamente indicar as condições para sua realização. Naquele contexto, portanto, caberia aos poderes públicos locais a escolha de como operacionalizar as orientações dadas, como ocorreu em Recife, conforme apresentamos a seguir. 


\subsection{PROGRAMA MANUEL BANDEIRA DE FORMAÇÃO DE LEITORES - PMBFL}

De acordo com Bandeira, Brandão e Rosa (2009), o Programa Manuel Bandeira de Formação de Leitores teve início em 2006 e visava promover ações voltadas ao fortalecimento de uma política de leitura na cidade do Recife, com o propósito de propiciar que a leitura e produção de textos se tornassem práticas cotidianas nas escolas municipais.

Foram definidas como ações a serem desenvolvidas pelo Programa: a criação de rede de leitores, a ampliação e diversificação dos acervos, a ampliação de professoras de biblioteca, a formação continuada de mediadores de leitura, contadores(as) de histórias Infantis, a constituição de uma linha editorial e a realização concurso para instalação ou reestruturação de bibliotecas. Considerando os objetivos desta pesquisa, esta última ação é a que iremos enfocar (BANDEIRA, BRANDÃO E ROSA ,2009).

Para a realização das reestruturações e implantações de bibliotecas escolares, foi lançado um edital que disponibilizava um limite de 40 projetos a serem aprovados. Em resposta, vinte e três escolas enviaram projetos para instalação e trinta e cinco enviaram projetos de reestruturação. Apesar do limite estabelecido, as cinquenta e oito escolas foram aprovadas pela gestão municipal e estavam aptas a receberem os recursos indicados no edital (BANDEIRA, BRANDÃO E ROSA ,2009).

\subsection{DIRETRIZES DA IFLA/UNESCO PARA A BIBLIOTECA ESCOLAR}

O objetivo destas diretrizes é auxiliar as escolas na implementação dos princípios expressos no manifesto: biblioteca escolar no ensino e aprendizagem para todos, publicado em 2005 pela IFLA/UNESCO. Além de dar suporte e orientação para a comunidade bibliotecária.

As diretrizes foram escritas por pessoas de diferentes países e em situações diversas, para atender às necessidades dos mais variados tipos de escolas. Por isso, é ressaltada no documento a importância de se considerar o contex to local ao ler o texto.

De acordo com as diretrizes da IFLA/UNESCO (2005) torna-se imprescindível as BEs possuir uma política própria que reflita os objetivos e a realidade da escola, ressalta-se ainda, que todos os membros da comunidade escolar devem se articular e se engajar tanto para a escrita da política, quanto para o alcance das finalidades estabelecidas na mesma. 


\subsection{BIBLIOTECA ESCOLAR COMO ESPAÇO DE PRODUÇÃO DO CONHECIMENTO: PARÂMETROS PARA BIBLIOTECAS ESCOLARES.}

Além dos documentos oficiais e de orientações advindas de órgãos internacionais como é a IFLA, também encontramos no Brasil documentos que indicam parâmetros de qualidade para bibliotecas escolares. Um estudo abrangente na área foi realizado pelo Grupo de Estudos em Biblioteca Escolar (GEBE), sediado na UFMG, (CAMPELLO et al, 2010).

O processo de formação do texto partiu da análise feita a partir dos dados de um estudo realizado com 685 escolas espalhadas em todo o país e teve como pilar para sua formulação, a noção de biblioteca escolar como espaço de conhecimento, ou seja: "As bibliotecas são espaços de aprendizagem que propiciam e estimulam conexões entre saberes; que são laboratórios - não de equipamentos e apetrechos - mas de ideias". (CAMPELLO et al, 2010 p. 7).

Foi discutido entre os membros da GEBE e posteriormente estabeleceu-se os parâmetros para identificar a qualidade da BE, os seguintes aspectos: espaço físico, acervo, computadores com acesso à internet, organização do acervo. Os indicadores apontam níveis a partir dos aspectos mencionados, categorizando as BEs desde um nível básico até o exemplar. O nível básico representa um ponto de partida, o patamar mínimo considerado essencial para uma biblioteca funcionar. O nível exemplar, por sua vez, representa uma meta a se alcançar. Estes instrumentos serviram de referência para a elaboração dos questionários e roteiros de entrevista utilizados na presente pesquisa, como detalhado no item que se segue.

\section{PROCEDIMENTOS METODOLÓGICOS}

Trata-se de uma pesquisa quali-quantitativa que teve como objetivo investigar as 58 bibliotecas escolares do Recife contempladas com instalação ou reestruturação pelo PMBFL.

Inicialmente foi feito um contato por telefone com todas as escolas, com o objetivo de verificar se as BEs estavam em funcionamento. Obtivemos, como dado neste primeiro momento, que quarenta e duas delas afirmaram estar com BE em funcionamento e dezesseis que não funcionavam mais, ou estão temporariamente desativadas. Sendo estas distribuídas conforme mostra o quadro:

Quadro 1: Levantamento das $58 \mathrm{BE}$ apoiadas pelo PMBFL quanto a seu funcionamento

\begin{tabular}{|c|c|c|}
\hline 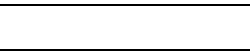 & Em funcionamento & Não Funcionamento \\
\hline Instalação & 14 & 8 \\
\hline Reestruturação & 28 & 8 \\
\hline
\end{tabular}

Fonte: As autoras 
O Quadro 1 nos revela um dado inicial relevante, ou seja, do total de 58 BEs, 16 não estavam funcionando, o que representa 28\%. Para compreender mais detalhadamente como era esse funcionamento das BEs, foram aplicados questionários ${ }^{1}$ e realizadas observações em dezesseis das instituições contempladas pelo Programa que afirmaram ter BE funcionando. Responderam aos questionários professoras, integrantes da equipe gestora e professoras que estavam lotadas nesses espaços. Das dezesseis escolas contempladas nesta análise, sete tiveram BE instaladas e nove reestruturadas com apoio da gestão municipal. Estas escolas foram escolhidas de forma que alcançassem as instituições das seis Regiões Político Administrativas (RPAs) e para que obtivessem dados de BEs instaladas e reestruturadas.

Para assegurar este critério geográfico, as escolas foram distribuídas em 11 grupos de acordo com sua proximidade e não necessariamente seguindo o critério de regionalização estabelecido pela Prefeitura da cidade e visitamos escolas conforme mostra a distribuição do Quadro 2, abaixo:

Quadro 2: Escolas que foram observadas e que responderam ao questionário

\begin{tabular}{|c|l|c|c|}
\hline \multicolumn{2}{|c|}{ Escolas visitadas } & Reestruturação & Instalação \\
\hline & & G1R & --- \\
\hline G1 & RPA 4 & G2R & G2I \\
\hline G2 & RPA 5 & G3I & G3R \\
\hline G3 & RPA 4, & G4I & G4R \\
\hline G4 & RPA 6 & G5R1, G5R2 --- \\
\hline G5 & RPA 4 & G6R & G6I \\
\hline G6 & RPA 1 & G7R & G8I \\
\hline G7 & RPA 6 & G8R & G9I \\
\hline G8 & RPA 2, 3 & --- & G10I \\
\hline G9 & RPA 3 & --- & \\
\hline G10 & RPA 2 & & \\
\hline
\end{tabular}

Fonte: As autoras

Como pode ser visto, foram dados códigos às escolas para preservar as identidades das instituições. Assim, se usará no decorrer do texto siglas que significam o grupo ao qual pertencem é uma escola com biblioteca reestruturada ou instalada. Para os casos em que houver mais de uma escola reestruturada ou instalada por grupo indicaremos a diferença com uma numeração sequencial (exemplo: G5R1, G5R2).

Esta etapa da pesquisa teve como objetivo verificar quais as condições de funcionamento nos aspectos físicos, materiais. De acordo com o Caderno de Educação Municipal (V.4, 2009), já mencionado acima, 58 escolas teriam sido contempladas pelo PMBFL. Porém, nesses dois primeiros momentos da 
pesquisa, obtivemos a informação de que pelo menos três escolas do total de instituições não chegaram a receber o valor do financiamento, devido a diferentes fatores. Assim, duas escolas (G8R e G8I), que estão no grupo das 16 bibliotecas observadas, afirmaram não ter recebido o repasse da Prefeitura.

Os motivos apresentados pelas escolas foram: G8R por não ter sua sede em um prédio alugado pela prefeitura e G8I por não possuir espaço adequado para a implantação de uma biblioteca. Estas informações foram fornecidas pelas gestoras das referidas escolas, e não foram confirmadas com a secretaria de educação da cidade do Recife. Mesmo considerando essa informação, optamos por incluir essas duas escolas em nosso estudo, considerando que elas informaram que tinham biblioteca em funcionamento e estavam na listagem oficial da secretaria de educação como integrantes do PMBFL.

Os resultados a seguir estão agrupados conforme os aspectos elencados nos Parâmetros criados pelo GEBE.

\section{CONDIÇÕES DE ESPAÇO FÍSICO E RECURSOS MATERIAIS}

Para esta discussão foram tomados como referência os seguintes aspectos: espaço físico, acessibilidade, adequação do espaço e condições de uso, recursos materiais - Acervo e equipamentos presentes nas 16 unidades escolares visitadas.

\subsection{ESPAÇO FÍSICO - ACESSIBILIDADE}

A biblioteca ser acessível a toda a comunidade escolar é um parâmetro de qualidade importante. O documento da IFLA/UNESCO (2005, p.8), fala da prioridade da BE se localizar no térreo em local central e de fácil acesso, além de atender às necessidades de pessoas com deficiência. Os parâmetros propostos pelo GEBE (2010, p.12) defendem como critério que: “A biblioteca escolar conta com espaço físico exclusivo, acessível a todos os usuários". Nesta questão, vimos que das 16 escolas analisadas, todas as BEs ficavam no térreo e 15 identificaram no questionário o trajeto feito para a chegada na biblioteca como acessível, exceto G10I. Entretanto, através das observações, percebemos que 13 tinham o trajeto realmente acessível, enquanto três (G10I, G3I e G8I) apesar de se localizarem no térreo, possuíam obstáculos que impossibilitavam o livre acesso de pessoas com deficiência. Outro fator percebido foi que o interior da BE, muitas vezes, não permite o livre acesso de pessoas com mobilidade reduzida devido ao espaço menor e a sua forma de ocupação. 


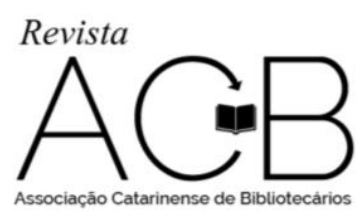

Revista ACB: Biblioteconomia em Santa Catarina, Florianópolis, v. 23, n. 1, p. 6-20, dez./mar., 2018.

Para entender esse fator foi feita a pergunta sobre o espaço interno das BEs possibilitarem a acessibilidade de pessoas com mobilidade reduzida. Neste item, 12 escolas afirmaram que seus espaços eram transitáveis, exceto G3I, G6I, G8I e G10I. Porém constatamos que apenas 7 BEs eram realmente acessíveis. O problema em G6I e G8I era a falta de espaço. Foram consideradas acessíveis em parte as bibliotecas G6R, G1R, G4R, G3I, G3R, G2I e G5R1 pois possuíam objetos bloqueando os caminhos, embora o espaço fosse amplo. As causas percebidas para este fator foram que quatro destas, estavam inacessíveis por causa das mesas interativas ${ }^{2}$ estarem ocupando grande parte do espaço das bibliotecas e as demais (G2I, G1R e G4R) poderiam ser melhoradas se os móveis fossem organizados de forma diferente.

Apesar da maioria das escolas terem uma preocupação de tornar o percurso acessível, percebe-se que ainda é necessário que haja a sensibilidade para que esses espaços sejam frequentados por toda a comunidade escolar. Uma avaliação de bibliotecas escolares no Brasil, feita pelo MEC em 2011 aponta que em várias unidades, a BE foi desmontada por necessidade de criação de salas de aula. No caso das escolas aqui analisadas, percebemos que a biblioteca também perde espaço diariamente para diversos fins.

\subsection{ESPAÇO FÍSICO - ADEQUAÇÃO DO ESPAÇO}

As diretrizes da IFLA/UNESCO (2005, p.8) ressaltam a necessidade de se oferecer iluminação e ventilação adequadas para o funcionamento desses espaços. Com relação à iluminação, 2 BEs (GR2 e G8I) se encontravam em condições ruins, 3 (G6I, G3I, G5R2) regulares e 11 ótimas. Essas informações foram confirmadas nas observações, conforme os informantes dos questionários.

No que se refere à ventilação, 2 escolas (G3I e G5R1) se identificaram em situação regular e 14 em situação ótima. Apesar disso, nas observações feitas, vimos que duas (G4R, G3I) estavam com a ventilação ruins, duas (G5R2, G2R) em estado regular e 12 em ótimo estado. A maioria possuía ambiente climatizado, algumas só possuíam ventiladores e janelas e as que estavam em situação ruim o arcondicionado precisava de manutenção.

Com relação ao tamanho, 5 escolas (G4R, G5R1, G5R2, G7R e G9I) avaliaram que seu espaço de biblioteca comportava uma turma de 35 alunos, contudo percebemos que apenas 4 comportariam este número de pessoas. As demais estavam dividias da seguinte forma:

\footnotetext{
${ }^{2}$ Mesas Educacionais da Positivo 
Quadro 3: Dimensões da BE

\begin{tabular}{|c|c|c|}
\hline Até 10 alunos & 11 a 20 alunos & 21 a 30 alunos \\
\hline 1 Escola= G6I & $\begin{aligned} 4 \text { escolas }= & \text { G3R, G1R, G8I } \\
& \text { e G3I }\end{aligned}$ & $\begin{array}{c}6 \text { escolas }=\text { G2I, G10I, G4I, G6R, } \\
\text { G2R, G5R1 }\end{array}$ \\
\hline
\end{tabular}

Fonte: As autoras

A maioria das bibliotecas não possui espaço para a realização de atividades individuais como, por exemplo, leitura de livros ou estudo. Apenas 5 (G7R, G5R2, G1R, G6I, G2R) disponibilizam ambiente para tais ações.

Sobre o tamanho das BEs, o documento da IFLA / UNESCO (2005) diz que o espaço deve ter, entre outras atribuições, flexibilidade para a realização de diversas atividades. Na maioria das bibliotecas não é possível, por exemplo, organizar rodas de leitura, sentar no chão e realizar atividades que necessitem de um maior espaço, inviabilizando assim, algumas atividades pedagógicas.

\subsection{ACERVOS}

Apenas uma biblioteca (G3R) não possuía livros para todas as faixas etárias atendidas na escola, contando apenas com livros didáticos. No que diz respeito ao quantitativo de livros as escolas se distribuem da seguinte forma:

Quadro 4: Quantitativo de livros por BE

\begin{tabular}{|c|c|c|c|c|}
\hline $\begin{array}{c}\text { Menos que 1 } \\
\text { livro por aluno }\end{array}$ & $\begin{array}{c}\text { Mais que 1 livro } \\
\text { por aluno }\end{array}$ & $\begin{array}{c}2 \text { a } 3 \text { livros por } \\
\text { aluno }\end{array}$ & $\begin{array}{c}4 \text { ou mais livros } \\
\text { por aluno }\end{array}$ & $\begin{array}{c}22,4 \text { livros } \\
\text { por aluno }\end{array}$ \\
\hline G3R & $\begin{array}{c}\text { G6I, G5R1, G7R, } \\
\text { G3I,G4R, G8I }\end{array}$ & G2I,G6R,G8R & $\begin{array}{c}\text { G4I, G10I, G1R, } \\
\text { G9I, G5R2 }\end{array}$ & G2R \\
\hline
\end{tabular}

Fonte: As autoras

De acordo com a Lei 12.244 de 2010 e também tendo como referência o documento do GEBE (p.13), uma BE deve ter um quantitativo mínimo de 1 livro por aluno. Já as diretrizes da IFLA/UNESCO dizem que uma coleção média de livros deve ter 10 exemplares por aluno. Apesar disso, uma das bibliotecas como pode ser visto, possui um acervo com um número inferior ao que dizem a lei e o GEBE. 


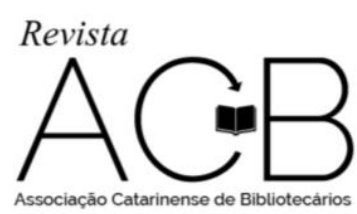

Revista ACB: Biblioteconomia em Santa Catarina, Florianópolis, v. 23, n. 1, p. 6-20, dez./mar., 2018.

Onze (11) escolas tinham todos os livros catalogados. Apenas duas (G2R e G4R) estavam com estado regular de conservação do acervo e os demais em bom estado. Para este ponto, na observação, foi levada em consideração a maioria do acervo.

Com relação aos outros tipos de acervo, todas as bibliotecas tinham mais que um tipo de material e possuíam dicionários, que são provenientes do Programa Nacional do Livro Didático (PNLD). No que se refere à hemeroteca, 50\% possuem gibis, exceto G1R, G2R, G2I, G3R, G4I, G4R, G6I e G8I. 75\% têm revistas, excluindo-se as escolas G1R, G2I, G3R e G4R. Além disso, havia jornal em 31\% das BEs, sendo elas G1R, G2R, G4R, G6R e G9I. Percebemos também a presença de jogos em 69\% das bibliotecas, excluindo-se apenas G2R, G3I, G5R2, G7R e G8I.

As diretrizes da IFLA/UNESCO (2005, p. 10) apontam a BE como "porta de acesso à atual sociedade baseada na informação e no conhecimento", devendo assim fornecer o acesso a computadores, equipamentos eletrônicos e audiovisuais.

Apenas duas BEs (G4R e G8I) não possuíam recursos de áudio e vídeo. As demais possuíam esses materiais, distribuídas da seguinte forma: $69 \%$ possuem TV, $62 \%$ DVD, $37 \%$ som, $31 \%$ data show e $25 \%$ microfone. Na questão de acesso a computadores, foi observado que 31\% tinham esse recurso, sendo que, $19 \%$ das bibliotecas apresentavam computadores só para professor de biblioteca, $12 \%$ para professor de biblioteca e alunos. 56\% não têm esse material. Nas bibliotecas que disponibilizavam computadores aos alunos a quantidade máxima encontrada foi três. E apenas $19 \%$ desses computadores tinham acesso à internet nos computadores.

\section{CONTINUIDADE DA POLÍTICA PÚBLICA IMPLANTADA POR MEOI DO PMBFL}

Para a compreensão da continuidade do PMBFL, realizou-se duas entrevistas semiestruturadas. Uma das entrevistas foi feita com a pessoa responsável atualmente pela coordenação do Programa e outra com uma das autoras do Programa. Além das entrevistas, com a mesma finalidade de compreender a continuidade do Programa, fizemos algumas perguntas abertas no questionário aplicado. Para preservar as identidades dos sujeitos entrevistados adotaremos a nomenclatura E1 (entrevistada 1) para uma das autoras do PMBFL e E2 (entrevistada 2) para uma das responsáveis pelo Programa.

O roteiro de perguntas feito para E1 teve como objetivo compreender o contexto de surgimento do Programa, verificando também as suas ideias e metas além de entender como aconteciam as realizações na prática dos objetivos e o papel das pessoas envolvidas neste processo. Já o roteiro de E2 tinha o objetivo de verificar quais as atividades desenvolvidas no Programa e quais as metas iniciais que o PMBFL tinha, permanecem acontecendo. 


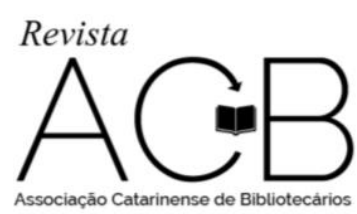

Revista ACB: Biblioteconomia em Santa Catarina, Florianópolis, v. 23, n. 1, p. 6-20, dez./mar., 2018.

De acordo com E1, para a realização das metas do Programa, foram feitas visitas para diagnosticar o estado que as BEs se encontravam. Isso pode ser visto neste trecho da entrevista: “[...] eu visitei com ela uma amostra pequena para a gente ter noção de como estava, né? fazer um diagnóstico [...] a gente percebeu que o quadro era crítico mas tinham algumas que se destacavam."

A partir das visitas realizadas por elas, foi constatado que o quadro de professoras de bibliotecas era precário, como pode ser visto no trecho abaixo: “[...] o que a gente encontrou na época foi: professoras readaptadas trabalhando na biblioteca, muitos professores que estavam em desvio de função, [...] estava em tratamento, estava de licença médica com problemas de voz [...]”.

Outra avaliação que E1 fez das bibliotecas foi sobre como se encontravam os espaços físicos. Falou que encontraram bibliotecas "feias", usadas como depósito de tudo que chegava na escola, "o que não tinha pra onde ir, ia para a biblioteca, aí virava um depósito de materiais de jogos, era mais almoxarifado[...]"

A partir destas percepções construídas nas visitas diagnósticas surgiram as ideias iniciais do PMBFL, que seria a melhoria do pessoal de biblioteca e na estética desses ambientes.

Aí a gente falou: a primeira coisa que a gente tem que fazer é, a biblioteca se tornar um espaço vivo [...] tem que ter uma professora [...] uma biblioteca pra funcionar bem precisa ter material humano bom, basicamente seria recurso humano que dá sentido a ela, mas a gente também que ria intervir física no ambiente $[\ldots]$

(Trecho da entrevista com E1)

A partir dessas duas metas surgiu a seleção para professores de biblioteca, os quais deveriam apresentar um projeto para a biblioteca e realizar uma avaliação. Também foi aberto um edital para que as escolas enviassem os projetos para melhoria ou implantação das BEs. Além deste edital, abriu-se uma linha editorial para incentivar a produção autoral de professores e estudantes.

Para compreender um pouco mais sobre a meta aqui analisada, perguntou-se se havia critérios para o recebimento do valor para reforma/instalação das BEs. E E1 respondeu que o primeiro critério seria ter o espaço, e que esses impedimentos eram de conhecimento das escolas e que mesmo assim, algumas enviaram os projetos. Sobre como as escolas investiram o valor recebido, E1 afirmou que parte do valor era destinado à compra de livros e que o PMBFL não interferia nas escolhas, a escola juntamente com suas comunidades, decidiam o que comprar.

Durante toda a entrevista, E1 ressaltava a importância de as políticas públicas levarem em consideração o desejo e as realidades de cada escola. E que a base do Programa era esta, trabalhar por 
"adesão", "a gente não queria também baixar um decreto, a gente queria também que a escola fizesse uma adesão

Confirmando esta proposta do PMBFL, E2 em entrevista falou de sua experiência enquanto professora de biblioteca na época de formulação do projeto de implantação da BE enviado ao Programa:

[...] a orientação do Programa era muito interessante porque solicitava que existisse a participação de todos os segmentos das escolas, [...] então a gente ouvia todo mundo para que fosse desenhado realmente a biblioteca que a gente queria, de que forma a gente queria essa biblioteca?

(Trecho da entrevista com E2)

E1 trabalhou no PMBFL por 5 anos e ressaltou que já em seu último ano que foi 2010, percebeu que "[...]teve muita mudança, em dois mil e dez eu percebi que havia quebrado espinha dorsal do Programa"

Ainda sobre a ruptura que houve no Programa, corroborando com o que disse a primeira entrevistada, E2 relatou que com a saída das pessoas que representavam "o Programa ficou acéfalo na época [...] nessa época o Programa ficou parado, a gente ficou sem formação". Falou ainda sobre a luta que os professores de biblioteca tiveram para permanecer trabalhando nelas.

A gente se reuniu para tentar ver o que podíamos fazer para não deixar o Programa morrer[...] aí os professores da biblioteca foram para a reunião, chegando lá a primeira coisa que a diretora de ensino disse foi o seguinte: eu não sei pra que a criança que não sabe ler vai pra biblioteca.

(Trecho de entrevista com E2)

Procurando compreender quais as atividades que o PMBFL desenvolve hoje, perguntamos a E2 se havia a possibilidade de um novo concurso para implantação/reestruturação de bibliotecas e ela respondeu que não, o que o Programa faz hoje são só as formações mensais, atividades dentro do Centro administrativo Pedagógico (CAP) que é onde se encontra a sede do Programa além de uma ação chamada “contando histórias e encantando escolas de biblioteca e fazer contação de histórias para algumas turmas.

Tentando compreender mais como se dão as relações das BEs contempladas com reestruturação/implantação, foi perguntado quais as dificuldades que enfrentavam para manter as bibliotecas em funcionamento antes do Programa. As respostas falavam sobre falta de espaço adequado para a biblioteca, falta formações e de professoras da biblioteca.

Quando responderam sobre as melhorias que o Programa ofereceu, apontaram a aquisição do espaço físico, a presença de professores de biblioteca, aquisição de mobiliário e recursos audiovisuais, livros e periódicos. Sobre isso G5R2 respondeu: "O acervo, a biblioteca ganhou vida, temos muita formação". 


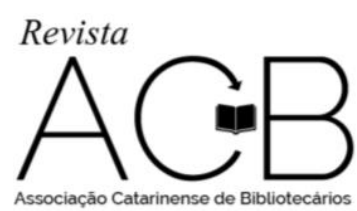

Revista ACB: Biblioteconomia em Santa Catarina, Florianópolis, v. 23, n. 1, p. 6-20, dez./mar., 2018.

Sobre as dificuldades encontradas hoje, foram apontados: falta de professores na biblioteca, necessidade de troca do mobiliário e de maior espaço por causa das mesas interativas que ocuparam espaço das BEs. G8R falou sobre a falta de mobiliário: “Que a PCR enviasse mobiliários, pois tem muitos livros em locais improvisados

\section{CONSIDERAÇÕES FINAIS}

O estudo buscou compreender as questões de funcionamento das BEs, investigando as condições de uso das bibliotecas, materiais e estruturais daquelas unidades que receberam apoio financeiro através do Programa Manuel Bandeira de Formação de Leitores.

Como primeiro dado, constatamos que $72 \%$ do total de bibliotecas disseram estar em atividade. Entretanto, buscamos identificar que tipo de funcionamento acontecia nesses espaços.

Percebemos que a biblioteca é um espaço vulnerável, pois o seu papel de produtora de conhecimento e formadora de leitores críticos por vezes parece não ser reconhecido pela comunidade escolar e pelos gestores públicos. Desta forma, vem perdendo seu espaço para outros fins como foram os casos das BEs que perderam parte ou todo o espaço para as mesas interativas, para guardarem caixas fechadas, sobras de livros didáticos, entre outros.

Consideramos, a partir do estudo realizado, que esse quadro se dá porque apesar de ter tido um grande investimento da prefeitura do Recife na política de formação de leitores implementada através do PMBFL, não houve uma continuidade nas metas e ações do Programa, mantendo-se somente a meta de formação para professores de biblioteca. Consequentemente, há uma falta de acompanhamento e de subsídios necessários para manter as bibliotecas desempenhando suas funções.

A presente pesquisa nos permitiu compreender como as BEs do Recife contempladas pelo Programa se encontram física e estruturalmente. Além de fornecer dados para compreender como o PMBFL contribui para esses espaços continuarem funcionando atualmente. A partir dos dados coletados é possível se aprofundar sobre o tema em novas pesquisas abrangendo questões administrativas, políticas, profissionais e pedagógicas da área. 


\section{REFERÊNCIAS}

BANDEIRA, Carmem; ROSA, Ester; BRANDÃO, Maria (Orgs). Programa Manuel Bandeira de Formação de Leitores: uma política de leitura na Rede Municipal de Ensino do Recife. Caderno Da Educação Municipal de Ensino do Recife. Vol. 4, Recife, 2009.

BRAGA, A.A; PAULA R.S.L; A biblioteca escolar e sua representação educativa: Cad. Ed. Soc., Inhumas, v.5, p.245-257,2014.

BRASIL. Decreto-lei n. ${ }^{\circ}$ 12.224, de 24 de maio de 2010. Dispõe sobre a universalização das bibliotecas nas instituições de ensino do País. Brasília/DF, 2010.

Brasil, Avaliação de Bibliotecas Escolares no Brasil. Ministério da Educação, Secretaria de Educação Básica, Brasília/DF, 2011.

CAMPELLO, Bernadete; et al. Parâmetros para Bibliotecas Escolares Brasileiras: fundamentos de sua elaboração I. Informação \& Sociedade: Estudos, João Pessoa, V. 21, n.2, p. 105-120, maio/ago. 2011.

CAMPELLO, Bernadete; et. al. Situação das bibliotecas escolares no Brasil: o que sabemos? In: Biblioteca Escolar em Revista, Ribeirão Preto, v. 1, n. 1. p. 1-29, 2012.

CAMPELLO, Bernadete; et. al. Pesquisas sobre biblioteca escolar no Brasil: o estado da arte. In: Revista eletrônica de biblioteconomia e ciência da informação. V. 18, n. 37, p. 123-156, 2013

Fundo Nacional de Desenvolvimento da Educação-FNDE. Programa Nacional Biblioteca da Escola (PNBE). Disponível em: http://www.fnde.gov.br/programas/biblioteca-da-escola/biblioteca-da-escolaapresentacao?highlight=YToxOntpOjA7czo0OiJwbmJlijt9. Acessado em: 07/07/2015

FERNANDES, Célia. Letramento Literário No Contexto Escolar. In:GONÇALVES, Adair; PINHEIRO, Alexandra (Org.) Nas trilhas do Letramento, Prática Formação Docente. Campinas/SP, Mercado das Letras, 2011.

IFLA/UNESCO. Diretrizes da IFLA / UNESCO Para a Biblioteca Escolar. Disponível em: http://www.ifla.org/files/assets/school-libraries-resource-centers/publications/school-library-guidelines/schoollibrary-guidelines-pt br.pdf Acesso em: 8 de Abril de 2015.

PENALOSA, Fernando. Princípios e métodos de seleção de livros Seleção e aquisição de livros: manual para bibliotecas. Washington, D. C: União Pan Americana, 1961

PEREIRA, Andréa Kluge. Biblioteca na Escola. Ministério da Educação, Secretaria de Educação Básica, 2006.

SILVA, J.R;TENORIO O. G.; Biblioteca escolar e a mediação de leitura: Uma proposta de fichas pedagógicas de registros de livros. Londrina, v. 3, n. 1/2, p. 197-212, jan./dez. 2014.

SILVA, Waldeck. Miséria da Biblioteca Escolar. 3. Ed - São Paulo: Cortez, 2003.

UFMG - GEBE. Biblioteca escolar como espaço de produção do conhecimento: parâmetros para bibliotecas escolares. Belo Horizonte, 2010.

VIANNA, H.M. Pesquisa Em Educação: A observação. Brasília: Plano Editora, 2003. 


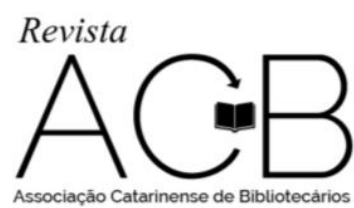

Revista ACB: Biblioteconomia em Santa Catarina, Florianópolis, v. 23, n. 1, p. 6-20, dez./mar., 2018.

\section{ALINE RAFAELA ANJOS}

Mestranda em Ensino das Ciências e Matemática pela Universidade Federal Rural de Pernambuco - UFRPE

E-mail: alinerafaela33@gmail.com

\section{STELA TAVARES BRANDÃO}

Graduada em Pedagogia pela Universidade Federal de Pernambuco- UFPE

E-mail: tavares.stela@gmail.com

\section{ESTER CALLAND ROSA}

Doutora em Psicologia Escolar e Desenvolvimento Humano - Universidade de São Paulo - USP

E-mail: esterosa2014@gmail.com 